\title{
Evaluación de la permeabilidad posterior a procedimientos endovasculares para pacientes con obstrucciones arteriales en miembros inferiores
}

\author{
Evaluation of permeability following endovascular procedures in patients \\ with lower limb arterial obstructions
}
Oswaldo Efraín Ceballos ${ }^{1}$, Jorge Adalberto Márquez², Juliana Messier ${ }^{3}$ Juan Pablo Charry4, Mauricio González ${ }^{5}$

\begin{abstract}
Instructor asistente, Departamento de Cirugía General y Vascular periférica, jefe Servicio de Cirugía Vascular Periférica, Fundación Universitaria de Ciencias de la Salud - Hospital de San José, Bogotá, D.C., Colombia

2 Instructor asistente, Departamento de Cirugía General y Vascular periférica, jefe Servicio de Cirugía General, Fundación Universitaria de Ciencias de la Salud - Hospital de San José, Bogotá, D.C., Colombia

3 Médica adscrita, Departamento de Cirugía General y Fellow de segundo año de Cirugía Vascular Periférica, Fundación Universitaria de Ciencias de la Salud - Hospital de San José, Bogotá, D.C., Colombia

4 Médico, residente de cuarto año, Departamento de Cirugía General, Fundación Universitaria de Ciencias de la Salud - Hospital de San José, Bogotá, D.C., Colombia

5 Médico, residente de segundo año, Departamento de Cirugía General, Fundación Universitaria de Ciencias de la Salud - Hospital de San José, Bogotá, D.C., Colombia
\end{abstract}

\section{Resumen}

Introducción. La enfermedad arterial periférica es una patología de difícil diagnóstico dado que su manifestación, en general, es asintomática, hecho que condiciona el avance significativo en su historia natural y el diagnóstico en estadio avanzado en la mayoría de los casos. Su prevalencia varía entre 3 y i8, mayor que la percibida por los profesionales de la salud, así como sus repercusiones clínicas, sociales y económicas, convirtiéndola en un importante problema de salud.

Materiales y métodos. Estudio de cohorte retrospectiva con pacientes llevados a procedimiento endovascular para manejo de obstrucciones arteriales en miembros inferiores entre febrero de 2010 y agosto de 20I6, en el que se evaluó la nueva obstrucción arterial en un seguimiento a un año. Se realizó un análisis de supervivencia no paramétrico a través del método de Kaplan-Meier para describir la frecuencia (tasa de incidencia e incidencia acumulada) de nueva obstrucción arterial.

Resultados. Ingresaron IO2 pacientes de I83 llevados a angioplastia, con edad promedio de 70,7 años (DE: 9,6) y predominio del sexo masculino con 56 pacientes (54,9\%). Durante el primer año de seguimiento, I8 pacientes (17,7\%) presentaron reestenosis (tasa de incidencia de 19,9 eventos por cada IOo pacientes al año). El mayor periodo de reestenosis fueron los primeros I20 días del seguimiento, con posterior estabilidad y presentación de mayor tasa de reestenosis nuevamente en los últimos I2o días del seguimiento, y en pacientes con intervención mixta (supra e infragenicular). Conclusión. La incidencia de reestenosis al año fue del $17,7 \%$, con permeabilidad superior al $80 \%$. Los procedimientos endovasculares son una estrategia segura que ofrece resultados similares a los reportados en la literatura. Palabras clave: enfermedad arterial periférica; arteria femoral; arteria poplítea; arterias tibiales; procedimientos endovasculares; permeabilidad.

Fecha de recibido: 30/11/2017 - Fecha aceptado: 5/06/2018

Correspondencia: Juan Pablo Charry, Carrera 64A \# 22 - 41, torre 2, apartamento 1304. Bogotá, D.C. Teléfono: 313-3297013. Correo electrónico: jpcharry@fucsald.edu.co

Citar como: Ceballos OE, Márquez JA, Messier J, Charry JP, González M. Evaluación de la permeabilidad posterior a procedimientos endovasculares para pacientes con obstrucciones arteriales en miembros inferiores. Rev Colomb Cir. 2018;33:280-4. https://doi. org/10.30944/20117582.73 


\begin{abstract}
Peripheral arterial disease is a pathology difficult to diagnose, since it mainly remains asymptomatic, which conditions the significant advance in its natural history and why at diagnosis it appears advanced in most patients. The prevalence of the disease has been determined in multiple studies, and varies from 3 to I8, higher than that perceived by health professionals, as well as its repercussions, both clinical, social, and economic. It constitutes a major health problem and remains an underestimated and underdiagnosed disease. Endovascular interventions of peripheral vascular pathologies are considered a fundamental treatment tool. However, there is no complete clarity regarding the patency time after endovascular interventions in the management of arterial occlusion, nor on methods that increase the degree of effectiveness of the procedure.
\end{abstract}

Key words: peripheral arterial disease; femoral artery; popliteal artery; tibial arteries; endovascular procedures; permeability.

\section{Introducción}

La enfermedad arterial periférica es una enfermedad de difícil diagnóstico, ya que su presentación es comúnmente asintomática, característica que condiciona el avance significativo en su historia natural y el diagnóstico en estadio avanzado en la mayoría de los pacientes. Su prevalencia ha sido determinada en múltiples estudios y varía entre 3 y I $8 \%$, ubicándose cerca del i6 \% en la población americana $^{\mathrm{I}, 2}$. Así mismo, se considera común en pacientes adultos y adultos mayores, estimándose aproximadamente en 500 a I.00o nuevos casos por año ${ }^{3,4}$. Su prevalencia es mayor que la percibida por los profesionales de la salud, así como sus repercusiones, tanto clínicas como sociales y económicas, lo que la convierte en un importante problema de salud. Es, por tanto, una enfermedad subestimada y subdiagnosticada ${ }^{5-7}$.

Los factores de riesgo relacionados con su desarrollo son clásicamente, la hipertensión arterial (HTA), el tabaquismo, la diabetes y la hipercolesterolemia ${ }^{7,8}$. Sin embargo, existen diferencias en la prevalencia de estos factores de riesgo según el territorio vascular analizado. La prevalencia de tabaquismo es mayor en pacientes con enfermedad arterial de los miembros inferiores respecto a otros territorios vasculares. $\mathrm{La}$ diabetes constituye otro factor de riesgo distintivo en la enfermedad arterial de los miembros inferiores, no solamente asociada con la presen- cia de obstrucciones, sino también con la predisposición a amputación y ulceración ${ }^{2,5}$.

Una de las herramientas más importantes en la búsqueda de diagnóstico temprano de enfermedad arterial periférica es el índice tobillo brazo (ITB) el cual es el método de elección para cribado en búsqueda de la enfermedad, además de ser el más costo-efectivo9. Hoy en día, las intervenciones por vía endovascular de patologías vasculares periféricas y centrales se consideran una herramienta fundamental en el tratamiento debido a los amplios beneficios y oportunidades en el enfoque de la patología arterial, ya sea por la minimización de riesgos anestésicos y quirúrgicos per se, así como también por la reducción de costos y tiempo de recuperación del paciente y menor tiempo de reincorporación a actividades rutinarias o laborales; adicionalmente, los procedimientos por vía endovascular han mejorado de manera significativa la supervivencia de los pacientes con patologías vasculares, tanto centrales como periféricas, en comparación con los procedimientos quirúrgicos $3,5,7,1 \mathrm{r}, \mathrm{II}$.

Sin embargo, conviene aclarar que en la actualidad no se cuenta con total claridad respecto al tiempo de permeabilidad posterior a intervenciones por vía endovascular para el manejo de oclusión arterial ni métodos que aumenten el grado de efectividad del procedimiento a largo pla$\mathrm{zO}^{12-14}$. Conocer este tiempo es el punto de partida para comparar posteriormente los procedimientos clásicos abiertos con aquellos realizados por vía 
endovascular para determinar cuál es la mejor opción para los pacientes. Por consiguiente, este estudio se propone evaluar la frecuencia y el tiempo de permeabilidad de las arterias de los miembros inferiores luego de una intervención por vía endovascular, utilizando una cohorte retrospectiva de pacientes que consultaron a una institución de cuarto nivel entre 2010 y 2016.

\section{Materiales y métodos}

Estudio de cohorte retrospectiva, en el que se estudiaron todos los pacientes que fueron llevados a procedimientos endovasculares para el tratamiento de obstrucciones arteriales en miembros inferiores en el Hospital de San José, desde el I $^{\circ}$ de febrero de 20IO, hasta el 3I agosto de 2016. Se excluyeron pacientes que no tuvieran datos de seguimiento después de la realización de la angioplastia. El criterio de ingreso a la cohorte fue la realización de la angioplastia de miembros inferiores, en tanto que el desenlace evaluado fue la permeabilidad primaria por reestenosis, a través de valoración clínica, estudios doppler, pletismografía o arteriografía. El tiempo de seguimiento fue un año.

En cada paciente se valoró, por medio de la historia clínica, el territorio al cual se le realizó angioplastia así como el tipo de angioplastia practicado, los factores de riesgo descritos en la literatura (diabetes, tabaquismo, hipertensión arterial y dislipidemia) y el desenlace con su respectiva fecha. La información fue recolectada por médicos especialistas en Cirugía vascular y Cirugía general en formación; el último seguimiento se hizo el 3i de agosto de 2017.

Las características demográficas y clínicas de los pacientes se describieron con frecuencias, medidas de tendencia central y de dispersión. Se realizó un análisis de supervivencia no paramétrico utilizando el método de Kaplan-Meier para describir la frecuencia (tasa de incidencia e incidencia acumulada) de obstrucción.

Estos análisis se llevaron a cabo en el paquete estadístico Stata I4.

Este estudio fue aprobado por el comité de ética e investigación con seres humanos del Hos- pital de San José. De acuerdo con la resolución 8430 de 1993 del Ministerio de Salud de Colombia, esta fue una investigación sin riesgo, pues se hizo a partir de la revisión de historias clínicas.

\section{Resultados}

De los pacientes que fueron tratados en el servicio de Cirugía vascular periférica en el Hospital San José, entre agosto de 2010 a 20I6, por enfermedad arterial periférica, se realizaron I83 procedimientos, de los cuales finalmente ingresaron al estudio I02; a 8I pacientes no fue posible realizarles el seguimiento, dado que no se contaba con las historias clínicas o registros de seguimiento posterior al procedimiento.

La edad promedio de los pacientes incluidos fue 70,7 años (DE: 9,6 años), con predominio de sexo masculino, en 56 pacientes (54,9\%). El 80 $\%$ de los pacientes tenía hipertensión arterial y el 56,9\% diabetes; el $48 \%$ tenía antecedente de tabaquismo; la mayoría no tuvo stent $(52,9 \%)$ o tuvo solo uno $(42, \mathrm{I} \%)$ y la principal localización anatómica de la obstrucción inicial fue la supragenicular (77,5\%). Las características basales de los pacientes se listan en la tabla I.

En los pacientes intervenidos se continuó la medicación indicada a su egreso: estatinas en $68,6 \%$, ácido acetilsalicílico en $67,7 \%$ y clopidogrel en $54,9 \%$.

Tabla 1. Características basales de los pacientes

\begin{tabular}{lcc}
\hline $\begin{array}{l}\text { Característica } \\
\text { Edad (años) }\end{array}$ & $\begin{array}{c}\mathbf{n = 1 0 2} \\
\text { Media 70,7 }\end{array}$ & $\begin{array}{c}\text { \% } \\
\mathbf{9 , 6}\end{array}$ \\
\hline Sexo & 56 & 54,9 \\
Masculino & 46 & 45,1 \\
Femenino & & \\
Comorbilidades & 82 & 80,4 \\
Hipertensión arterial & 58 & 56,9 \\
Diabetes & 49 & 48 \\
Tabaquismo & & \\
Localización & & \\
Infragenicular & 29 & 28,4 \\
Supragenicular & 79 & 77,5 \\
Mixto & 16 & 15,7 \\
\hline
\end{tabular}


Durante el primer año de seguimiento, I8 pacientes (I7,7 \%) presentaron estenosis (figura I); la tasa de incidencia de reestenosis fue de 19,9 eventos por cada Ioo pacientes al año; el mayor periodo de reestenosis correspondió a los primeros I2O días del seguimiento, con estabilidad posterior y mayor tasa de nueva reestenosis en los últimos I20 días del seguimiento.

De acuerdo con la localización anatómica de la obstrucción inicial, la reestenosis tuvo mayor presentación en los pacientes con compromiso de localización mixta, 6 de I6 pacientes (37,5\%), seguido por el territorio infragenicular en 3 de I9 pacientes ( $15,6 \%$ ) y supragenicular en 9 de 68 pacientes (I3,2\%) (tabla 2).

\section{Discusión}

La enfermedad arterial periférica ocupa un lugar importante dentro de las patologías que más afectan al ser humano. En la población mundial existe una prevalencia entre el 3 al I8 \% ${ }^{3-5,15}$. El manejo puede ser médico, quirúrgico o endovascu$\operatorname{lar}^{16}$. Para determinar cuál es la mejor estrategia de tratamiento es necesario describir y comparar la frecuencia de reestenosis entre las mismas; este estudio se propuso describir la incidencia acumulada y la tasa de incidencia de reestenosis en una institución de cuarto nivel de Bogotá.

En la población analizada de manera retrospectiva, se identificó que la edad de los pacien-

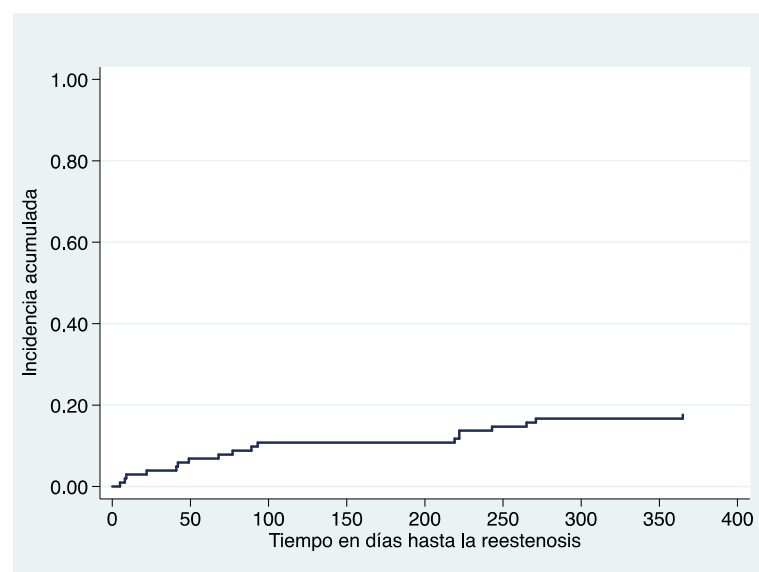

Figura 1. Incidencia acumulada de reestenosis estimada por el método de Kaplan-Meier tes en el momento de intervención, así como la presencia de comorbilidades identificadas en la literatura mundial es muy similar, con una media cercana a la séptima década de la vida y predominantemente en el sexo masculino ${ }^{\mathrm{I4,15}}$. Así mismo, se observó mayor distribución del compromiso inicial en territorio vascular con nuevos episodios de estenosis presentados en mayor cantidad en pacientes con compromiso mixto (supra e infragenicular), seguido por los de compromiso infragenicular, con similares resultados respecto a las publicaciones de la literatura ${ }^{\mathrm{I}}$, en las que, según Ruby, se presenta desde un 37 a $50 \%$ de estenosis en el infrapoplíteo. El determinante final de aparición de reestenosis se presentó en menor frecuencia respecto al hallado en la literatura actual ${ }^{1,4}$, a excepción de la tasa reportada por Siracuse ${ }^{\mathrm{I}}$, con poblaciones de características similares a la estudiada, logrando obtener un porcentaje libre de reestenosis mayor al $80 \%$ dentro del año de seguimiento evaluado.

Dada la naturaleza de cohorte retrospectiva de este estudio, hay limitaciones en la calidad de la información puesto que fue tomada a partir de la historia clínica, así también las hay debido al cambio de sitio de valoración de los pacientes, asignado por parte de las entidades prestadoras de salud, lo cual ocasiona pérdida de pacientes por seguimientos extrainstitucionales; de igual forma, se identificó que parte de los pacientes con mejores resultados con recuperación rápida de su funcionalidad, tornándose asintomáticos, en los primeros controles no continuaron en el seguimiento debido a la sensación de bienestar.

A pesar de las limitaciones de los estudios retrospectivos, el menor tiempo de seguimiento y

Tabla 2. Reestenosis por territorio vascular

\begin{tabular}{lccc}
\hline & \multicolumn{3}{c}{ Estenosis } \\
\cline { 2 - 4 } Territorio & $\begin{array}{c}\mathbf{S i} \\
\mathbf{n}(\%)\end{array}$ & $\begin{array}{c}\text { No } \\
\mathbf{n}(\%)\end{array}$ & Total \\
\hline Supragenicular & $9(13,2)$ & $59(86,8)$ & 68 \\
Infragenicular & $3(15,6)$ & $16(84,4)$ & 19 \\
Mixto & $6(37,5)$ & $10(62,5)$ & 16 \\
\hline
\end{tabular}


el número de pacientes recolectados en este estudio, se debe destacar la importancia del análisis realizado en este trabajo dada la escasa cantidad de reportes locales. Así mismo, es un referente inicial para la evaluación de la permeabilidad de las angioplastias practicadas en nuestra población, el cual permitirá calcular tamaños de muestra para investigaciones prospectivas y analíticas, de manera que se pueda estimar con mayor precisión la incidencia de reestenosis y se pueda comparar entre estrategias de manejo.

A partir de los resultados obtenidos, se propone realizar un estudio prospectivo que permita determinar cuáles son las comorbilidades que más se asocian a la reestenosis de vasos arteriales, posterior a procedimientos endovasculares, y si se presenta menor reestenosis y mejores resultados en procedimientos endovasculares frente a cirugía abierta.

\section{Referencias}

I. Lo RC, Darling J, Bensley RP, Giles KA, Dahlberg SE, Hamdan AD, et al. Outcomes following infrapopliteal angioplasty for critical limb ischemia. J Vasc Surg. 2013;57:I455-63.

2. Selvin E, Erlinger TP. Prevalence of and risk factors for peripheral arterial disease in the United States: results from the National Health and Nutrition Examination Survey, I999-2000. Circulation. 2004;1I0:738-43. doi Io.II6I/oI.CIR.ooooI379I3.26087.Fo.

3. Norgren L, Hiatt WR, Dormandy JA, Nehler MR, Harris KA, Fowkes FGR, et al. Inter-Society Consensus for the management of peripheral arterial disease (TASC II). Int Angiol. 2007;26:82-I57.

4. Muir KB, Cook PR, Sirkin MR, Aidinian G. Tibioperoneal occlusive disease: a review of below the knee endovascular therapy in patients with critical limb ischemia. Ann Vasc Surg. 20I7;38:64-7I.

5. Wu R, Yao C, Wang S, Xu X, Wang M, Li Z, et al. Percutaneous transluminal angioplasty versus primary stenting in infrapopliteal arterial disease: a meta-analysis of randomized trials. J Vasc Surg. 20I4;59:I7II-20.
6. Wiseman JT, Fernandes-Taylor S, Saha S, Havlena J, Rathouz PJ, Smith MA, et al. Endovascular versus open revascularization for peripheral arterial disease. Ann Surg. 2017;265:424-30.

7. Martínez J, Díaz JJ, Luján VP, Fernández MR, Ramírez E. Enfermedad oclusiva aortoilíaca o síndrome de Leriche. Rev Colomb Cir. 2017;32:214-22. doi: IO.30944/20II7582.27

8. Wölfle KD, Bruijnen H, Loeprecht H, Rümenapf G, Schweiger H, Grabitz K, et al. Graft patency and clinical outcome of femorodistal arterial reconstruction in diabetic and non-diabetic patients: Results of a multicentre comparative analysis. Eur J Vasc Endovasc Surg. 2003;25:229-34.

9. Salisbury AC, Li H, Vilain KR, Jaff MR, Schneider PA, Laird JR, et al. Cost-effectiveness of endovascular femoropopliteal intervention using drug-coated balloons versus standard percutaneous transluminal angioplasty. JACC Cardiovasc Interv. 20I6;9:2343-52.

Io. Alonzo DC, Atallah DA, Brescacin DL, Díaz DF, Tammer DI. Consenso de enfermedad vascular periférica. Rev Arg Cardiol. 2015;83(Suppl.3):46I-86.

II. Jiménez CE. Tratamiento endovascular del trauma vascular periférico. Rev Colomb Cir. 2012;27:290-7.

I2. Asadi H, Lee RJ, Sheehan M, Thanaratam P, Lee DM, Lee AM, et al. Endovascular therapy research in lower limb peripheral arterial disease published over a 5-year period: who is publishing and where? Cardiovasc Intervent Radiol. 20I7;40:343-50.

13. Sigvant B, Wiberg-hedman K, Bergqvist D, Rolandsson $\mathrm{O}$, Andersson B, Persson E, et al. A population-based study of peripheral arterial disease prevalence with special focus on critical limb ischemia and sex differences. J Vasc Surg. 2007;45:II85-9I.

I4. Siracuse JJ, Van Orden K, Kalish JA, Eslami MH, Schermerhorn ML, Patel VI, et al. Endovascular treatment of the common femoral artery in the Vascular Quality Initiative. J Vasc Surg. 20I7;65:1039-46.

I5. Saqib NU, Domenick N, Cho JS, Marone L, Leers S, Makaroun MS, et al. Predictors and outcomes of restenosis following tibial artery endovascular interventions for critical limb ischemia. J Vasc Surg. 2013;57:692-9.

I6. Singh GD, Armstrong EJ, Yeo KK, Singh S, Westin GG, Pevec WC, et al. Endovascular recanalization of infrapopliteal occlusions in patients with critical limb ischemia. J Vasc Surg. 2014;59:1300-7. 